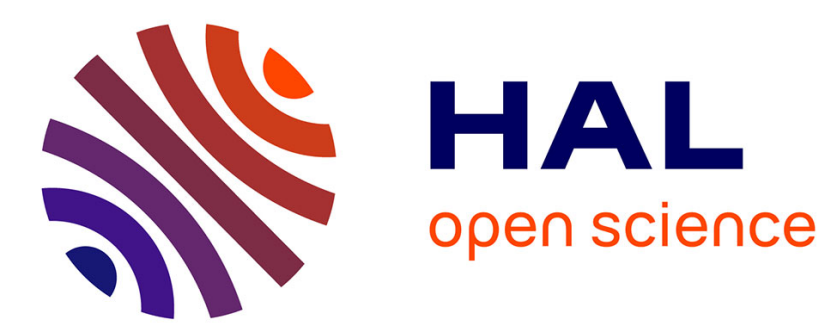

\title{
L'Indécidabilité de genre ( gender ) dans le premier roman du cycle de Marie, de Jean-Philippe Toussaint
}

Marie-Françoise Berthu-Courtivron

\section{To cite this version:}

Marie-Françoise Berthu-Courtivron. L'Indécidabilité de genre ( gender ) dans le premier roman du cycle de Marie, de Jean-Philippe Toussaint. Contemporary French and Francophone Studies, 2017, 21 (2), pp.169-180. 10.1080/17409292.2017.1343013 . hal-02187350

\section{HAL Id: hal-02187350 \\ https://hal.univ-rennes2.fr/hal-02187350}

Submitted on 17 Jul 2019

HAL is a multi-disciplinary open access archive for the deposit and dissemination of scientific research documents, whether they are published or not. The documents may come from teaching and research institutions in France or abroad, or from public or private research centers.
L'archive ouverte pluridisciplinaire HAL, est destinée au dépôt et à la diffusion de documents scientifiques de niveau recherche, publiés ou non, émanant des établissements d'enseignement et de recherche français ou étrangers, des laboratoires publics ou privés. 


\section{L'indécidabilité de genre (gender)}

\section{dans le premier roman du cycle de Marie, de Jean-Philippe Toussaint}

Article publié dans Contemporary French and Francophone Studies, volume 21, n 2, 2017, p. 169-180

\section{Marie-Françoise BERTHU-COURTIVRON, Univ Rennes (CELLAM)}

Derrière une intrigue assez courte et apparemment inconsistante, des personnages qui n'ont rien d'héroïque, une narration globalement linéaire, le roman de Toussaint concentre en fait un des formidables enjeux de notre société contemporaine: une remise en cause des catégories traditionnelles du genre, une confrontation inédite et parfois violente du masculin et du féminin, décloisonnés de leur binarité ancienne et réincorporés, au-delà des sexes, en des individus nantis de potentialités existentielles nouvelles, qui ne sont pas plus heureux pour autant.

\section{Les gages apparents d'une féminité univoque}

A première vue dans Faire l'amour, les catégories de genre semblent stables et la femme en titre donne toutes les garanties extérieures de sa condition : sa série de prénoms - Marie, Madeleine et Marguerite - redondante à l'initiale, crée à elle seule une première saturation du féminin, comme si c'était dérisoirement un collectif de femmes que Marie était censée représenter.

Cette héroïne est en outre féminine par sa gestuelle sensuelle, sa façon fréquente de « laisser tomber sa tête » sur l'épaule du narrateur «avec beaucoup de féminité et d'abandon » (61): c'est dans cette position qu'elle est prise en photo contre lui, «la tête légèrement inclinée sur [s]on épaule » (129). La pose sexuelle n'en est pas moins étudiée et convenue lorsque Marie s'offre aux regards, « le corps dénudé en travers du lit, les yeux ceints » d'un bandeau (33), scène d'anthologie érotique.

Marie correspond également au modèle social du genre par une propension exemplaire aux larmes. Quand le narrateur s'interroge sur son prénom Marie Madeleine dont il dit ignorer l'origine, il n'en cache pas la valeur symbolique, d'origine implicitement biblique : « elle ne l'avait pas volé, personne n'avait comme elle [...] ce don inné des larmes » (46). Indice extérieur d'une féminité vulnérable, la fréquence des larmes, trop abondantes pour être anodines, invite à s'interroger sur leur motif réel. Si la requête incessante d'être embrassée (17 et 73) reste insatisfaite, le narrateur superpose toute une série de souvenirs proches ou lointains de Marie en larmes (12), qui augmentent encore l'effet de son refus présent : c'est dire que le récit verse et mélange des larmes d'origines variées, de joie ou de frustration, donnant ainsi l'impression d'un comportement uniforme de Marie à tous les stimuli masculins. Cette preuve hyperbolique de féminité semble 
manipulée par le narrateur qui les regarde ou les fait couler non sans jouissance comme si cette liquidité, se substituant à celle qui lui fait défaut, le réconfortait dans son être.

Car comment expliquer le refus empêtré et pourtant obstiné du narrateur de ne pas donner le baiser ou la jouissance attendus si ce n'est de voir Marie couler un peu plus ? Ou d'avancer l'hypothèse de leur rupture, alors qu'il entretient le manque de crédibilité de ce motif en s'abstenant délibérément de lui donner une cause rationnelle, comme si la rétention d'information (il dit préférer se taire sur ce motif, 22) s'ajoutait à ses autres défections ? Ainsi la rupture semble défier la vraisemblance romanesque : pourquoi quitter Marie alors qu'elle lui manque dès qu'il est loin d'elle $(131,136)$ et puisque «l'absence de l'autre » les « rapproch[e]» (22) ? Tout le ressort du roman semble tenir dans cette aberration de légitimer une rupture par le simple fait d'en scander le leitmotiv dans le texte.

L'ambiguïté du narrateur et sa marge d' «indécidabilité » (145) se perçoivent à ses réponses inconsistantes (17) : « je ne pouvais pas lui dire maintenant que je ne voulais ni l'embrasser ni ne pas l'embrasser» 73). Se délectant de cet entre-deux, il se soustrait d'abord au rôle social du séducteur, quitte à passer pour sadique. Car ce que Marie veut, ce qui la mine pendant tout le roman de ne pas avoir, c'est un baiser-spectacle qui les replacerait chacun dans un rôle sexué clairement différencié et socialement établi où c'est l'homme qui prend l'initiative. D'où l'absurdité apparente d'une dispute récurrente, mais aussi d'une ténacité inexpliquée de l'homme à ne pas gratifier l'autre sexe de ce cliché libérateur. De fait, en se refusant, certes il garde le pouvoir, mais c'est lui qui fait la femme. Aussi Marie pleure-t-elle simplement le sexe masculin qui se dérobe, ou un cliché du genre auquel elle n'a plus accès ? Si le narrateur pratique l'esquive incessante du baiser de paix qui replacerait l'autre dans un cliché évident (et même parodique) et la rassurerait sur son identité héritée, c'est que cette tension entretenue artificiellement est essentielle : en n'embrassant pas, il la sort du cliché de la féminité passive en attente de reconnaissance, et il la contraint à une autre activité féminine (lacrymale) plus autonome, qui transfère la jouissance du partenaire sur la contemplation de la liquidité produite. Il la déplace ainsi dans une autre tradition de féminité, d'ascendance plus archaïque et ancienne, sublimant le sexuel (Marie-Madeleine qui épuise son Seigneur de sa gestuelle hyperbolique et de ses larmes). Déjà, il la force à dépasser les facilités contemporaines du genre et à se spiritualiser en remontant à sa source.

Le narrateur a tout intérêt à ne pas entrer dans le jeu, aussi flatteur soit-il : ce baiser le pousserait dans ses derniers retranchements, autant dire dans des tranchées collectives où il ne se reconnaît pas. 
Sur le plan professionnel, Marie travaille dans la mode, espace où s'extrêmisent l'incarnation du destin féminin à travers les robes qu'elle crée et le formatage des femmes à l'aune des valeurs marchandes. En renouvelant la forme mais non le fond, elle est l'inspiratrice directe du système puisqu'elle propose en imitation aux femmes des modèles de luxe. Elle porte trace implicite de cet impératif de mimétisme à travers ses «huit valises métalliques rembourrées et quatre malles identiques » (19) qui sont détentrices d'une surabondance identitaire (140 kilos de bagages, dont l'excédent est payé « rubis sur l'ongle», cliché humoristique du genre). Une fois sorties des valises, les robes reçoivent de l'écriture une étrange animation puisqu'elles paraissent dans la chambre «parées, altières, décolletées, séductrices » (20) : une fois accrochées par Marie, elles environnent le couple, «pendues aux battants des armoires», ou «alignées sur les deux portants de voyage » (20), et dans cette position où elles semblent tenir debout toutes seules, elles reçoivent par-delà leur diversité de couleur un même rôle assigné : elles deviennent par glissement métonymique la figure des corps qu'elles contiendront, vides de substance propre, réduits à leur forme décidée par la mode. C'est ainsi que le narrateur les imagine d'abord «en représentation », puis « comme dans une loge de théâtre improvisée » (20), prêtes pour leur future fonction de donner aux femmes une épaisseur existentielle dans un monde qui les façonne. Dans une société où la femme est invitée à une «performance » incessante ${ }^{1}$, ces robes sont chargées par l'écriture d'une intention qui les dépasse et alors qu'elles sont dites par l'auteur « désincarnées » (20), privées - à ce stade encore - de la chair des femmes, elles «semblent faire cercle autour » du corps «à moitié dénudé, et las » (20) de Marie, elle-même désemplie de son être, corps vide au milieu de ses reflets, de ces images effeuillées d'elle, comme autant de costumes faussement animés qui semblent avoir déjà commencé la comédie sociale pour laquelle ils ont été pensés.

Bientôt, ces défroques luxueuses sont soumises à un fantasme de mort, et visualisées par le narrateur comme un « cortège figé », robes « languissantes qui avaient pris formes humaines dans la pénombre et pendaient », «suppliciées, aux gibets de fortune des portants de voyage » (33). L'effet de personnification qui provoque la peur du narrateur vient conforter la mise en scène de théâtre où les rôles conçus par la mode insufflent aux femmes (encore virtuelles, même si le corps de l'unique femme présente, Marie, sert d'emblème) une identité d'emprunt qui leur est mortifère. Marie ellemême est contaminée lorsqu'elle se laisse tomber sur le lit, «donnant dès lors à sa silhouette des allures de star énigmatique », au milieu de ses robes froissées : elle semble rattrapée non seulement par le destin médiatisé des femmes, inhérent à la mode, mais par le danger de mort lié à cette performance du genre, «figure vaincue et ophélienne dans son lit mortuaire » (24). Fantasme récurrent puisque peu avant, Marie avait dû déclarer à la douane sa «création récente », entourée d'un « linceul », corps « purement virtuel, éviscéré » (84). 
Vidé de son contenu propre, le corps des femmes est littéralement momifié par la mode : à la fin du roman le narrateur revoit dans le musée (espace où sont conservés hors du temps les destins figés) les créations de Marie, des mannequins debout, «statues pétrifiées » (144).

Comme par ratification de ce destin commun qui leur est tragiquement funeste, Marie va enfiler bientôt une de ses créations, ce qui est présenté par le narrateur comme tout à fait exceptionnel et un mauvais présage (48). Faut-il d'ailleurs voir un lien entre le fait que dans ce début Marie est déclarée « incomparable » (51) et le fait qu'elle ne porte jamais les robes proposées en parures aux autres représentantes de son sexe ? Comme surpris par cette incarnation spontanée de la femme s'identifiant au concept prévu de la féminité, il est symptomatique que le narrateur s'étonne alors de sa présence par une question («Tu es là ? 48) qui semble, dans une polysémie suggestive, remettre en cause le fondement de son être comme si elle était un simulacre, une sorte de pantin du genre, dont la légitimité était devenue irréelle.

\section{Les ressorts d'une virilité affirmée}

De fait, cette féminité alibi est compensée par une virilité de Marie dans la sexualité comme dans l'art. Son nom la place d'ailleurs sous ces doubles auspices, car si les prénoms sont féminins, le patronyme (de Montalte) est doublement masculin, hérité non seulement du père mais de Blaise Pascal (dont elle porte le pseudonyme littéraire). Outre cette surcharge onomastique, l'ascendance aristocratique crée un poids additionnel transmis par les hommes et ancré dans l'Histoire, avec lequel il sera difficile au narrateur privé de nom de rivaliser. Ce patronyme de Marie est trop élaboré pour être innocent : véritable concept, il réunit tant les sexes que les classes sociales et les époques, en un creuset révélateur d'une humanité totale mais peut-être, de ce fait, vouée à la confusion et à la quête de soi.

Cette virilité de Marie s'exerce en particulier dans le domaine sexuel. Sous la pulsion du désir, elle sait «d'un geste autoritaire, sûr et précis » «s'empar[er] de la main » du narrateur et « resserr[er] les cuisses autour de sa prise » (25) ou plus violemment «enfermer » le sexe de son amant «dans l'étau de ses cuisses » (29). Cette femme que le narrateur qualifie de «culottée » (19) sait aussi le traiter de «mauviette » et lui prêter son manteau pour qu'il n'ait pas froid dans la nuit de Tokyo (50-51).

Dans le domaine artistique, Marie est doublement virile, autant dans sa réception sociale que dans sa création esthétique. Elle sait en effet «imposer le silence et le respect nécessaires à sa concentration » à un aréopage d'hommes qui la suivent «à distance », qui «n'os[ent] l'aborder », « intimidés » par sa «présence forte, déterminée » (100). Mais elle s'est attribué aussi une force prométhéenne dans sa conception de l'art, «imprévisible » (51) et inclassable, cet art « légèrement dingue » (50) comme elle, et parfois dangereux dans son concept même, quand il oublie qu'il doit 
se mesurer au réel. Elle reste pionnière dans son défi à la raison se heurtant aux limites d'une logique pratique : avant même les robes en sorbet ou en miel que l'on verra dans les romans suivants, les deux matériaux avec lesquels elle crée ses robes sont ici le «titane » et le «Kevlar» (19), connus tous les deux pour leur résistance exceptionnelle à la rupture, plus forte que celle de l'acier (le Kevlar étant même utilisé dans les gilets pare-balles), ce qui met en question l'adéquation de ces fibres au contexte délicat de la mode féminine.

Cette dualité de Marie se retrouve projetée et comme démultipliée sur des motifs annexes, entretenant le caractère irréductible du personnage : ainsi son humeur «fantasque » (51) se retrouve dans l'inexplicable instabilité thermique de la chambre «tantôt surchauffée et tantôt glacée »: Marie se plie aux «caprices » et aux «dérèglements » du thermostat «fantasque » (135), comme le narrateur est censé se soumettre aux siens. Invitée à se prononcer sur sa priorité, la faim ou le froid, elle ne tranche pas : «froid et faim » dit-elle (53); du thé ou du café ? «elle m'avait dit les deux » (23). Cette impossibilité de renoncer à la dualité est symptomatique d'une « confusion regrettable » (57) qui s'attache à tous ses actes et la rend «tuante » (51), puisqu'elle fait « tourner sur [lui]-même à toute vitesse » le narrateur «jusqu'à ce que [s]es instruments s'affolent » (136) : serait-ce le sens à donner à cette « vérité » de Marie présentée en titre du troisième roman et jamais explicitée ?

Alors, qui sait si Marie ne pleure pas sur cette féminité que l'auteur lui a retirée, sur cette hybridité qu'il lui affuble et qui la laisse pantelante et ahurie, sinon ahurissante, toujours ailleurs que là où on l'attend? en tous cas par son «outrance » (88), dans un au-delà d'une norme sociale de la modération, apanage des femmes. Cette dualité de Marie reste donc non résolue, en suspens dans le roman, comme un mur transparent auquel se heurte le narrateur et qui semble en fait le seul motif plausible de rupture.

Cette contradiction intrinsèque vient ainsi puiser dans une double inversion des catégories de genre, puisque par une répartition nouvelle, comme par un effet de vases communicants, les attributs traditionnels de chacun des deux sexes sont reportés sur l'autre.

\section{L'épuisement du masculin}

Toutes ces contradictions sous-jacentes déstabilisent le narrateur qui se retrouve lui-même privé des normes sociales de la virilité. Il ne s'affirme qu'en maître de l'esquive, réfractaire au rôle du séducteur qui devra attendre le troisième roman pour être assumé par Jean-Christophe de G. La virilité ayant été détournée par Marie dans le roman, c'est à une partie de lui-même qu'il est confronté face à elle, et qu'il lui est impossible d'étreindre.

Face à l'esprit créateur débridé de Marie, le narrateur reste donc impuissant et désœuvré. Ses deux prérogatives traditionnelles, sociale et sexuelle, lui sont retirées. Non seulement il ne travaille 
pas, mais c'est en toute lucidité que, «sans statut, dans son ombre », il accompagne l'artiste en tournée, ayant accepté explicitement son rôle de «cortège », d'« escorte » (23), de «Monsieur Thatcher» (91). S'il analyse les «arrière-pensées légèrement perverses » (22) qu'a eues Marie en lui proposant de l'accompagner au Japon et s'il conclut qu'elle a voulu jouer de sa position dominante, jamais il ne donne la raison de sa propre acceptation (si ce n'est prendre à revers Marie, persuadée qu'il va refuser) alors que lui-même, pourtant conscient des enjeux, assume sans état d'âme apparent sa fonction sociale subalterne.

Ce double transfert des attributs sexués traditionnels se concrétise par une errance à deux dans les rues de Tokyo où est mise en scène une inversion spectaculaire des rôles sociaux dans le travestissement. Conformément à la mascarade dans le jeu social pointée par Butler², le couple investit ici une panoplie désaxée du genre. Par-delà le cliché (en plus de porter une de ses robes « de collection, à vingt mille dollars », Marie incarne à nouveau l'idéal féminin par une gestuelle très câline et «se blotti[ $\mathrm{t}]$ contre » le narrateur - 51), ce dernier se plaît à se décrire non seulement dans un attirail pitoyable et peu viril («les pieds rougis de froid dans [s]es fragiles sandales » 60), mais ayant endossé, pour lutter contre le froid, le manteau de Marie «beaucoup trop petit pour [lui]» (51). Le parti pris d'autodérision, constant dans le roman, renforce délibérément le décalage (plus tard, en se montrant avachi «dans [s]on caleçon fané », le narrateur insistera sur son allure déplorable dans une parenthèse sapant, en-deçà du discours, la figure d'autorité : « (quel homme d'action, vraiment) » 125).

Il y aurait donc une forme de jouissance chez le narrateur à être dans cette position socialement dévalorisante et à prendre le modèle à contre-courant, à faire trembler les catégories de genre : d'où le parti pris d'accentuer le ridicule dans la description de cet homme travesti («moi empêtré dans un manteau en cuir quatre fois trop petit pour moi qui me remontait au milieu des bras » 53). Le travestissement réciproque vulgarise non seulement l'image du sous-homme, mais celle de la sur-femme (mannequin ou cliente potentielle du défilé de mode). Plus que toute théorie sur la subversion des identités, la mise en scène littéraire de cette fluctuation par rapport à la norme sociale, de ce trouble jeté dans le genre est ici des plus jubilatoires. De fait, il est intéressant qu'elle s'accompagne dans le texte d'une des rares notations de partage sentimental: les amants sont décrits légers, «courant la main dans la main »(51). C'est donc quand le couple en pleine conscience joue sa propre mascarade en abîme de la mascarade sociale que, libéré des injonctions du genre, il s'apaise.

C'est au contraire quand la pression sociale se renforce et quand le narrateur est soumis aux attentes liées à son rôle que le pacte est rompu et que le couple se déchire. Son échec à trouver un taxi prend la tournure d'un drame existentiel : d'abord « éconduit » par un premier taxi, le narrateur 
«[f]urieux et impuissant» se met à héler un autre taxi «n'importe comment, sans conviction» (66). Il refuse d'entrer dans une saine et virile émulation pour sauver une compagne qui a froid dans l'archaïsme de la nuit : comme par un effet de cette contagion qui fonde les rapports sociaux, les taxis ne peuvent l'apercevoir. Cette invisibilité par l'autorité (le chauffeur de taxi représentant l'instance sociale qui a le pouvoir de décision et ne réagit qu'aux sollicitations spectaculaires du genre) est suivie d'un désaveu immédiat de la femme, « exaspérée de [s]on inefficacité » (66) : ces deux réactions, d'ignorance et de colère, sanctionnent le refus du narrateur d'assumer une binarité rassurante des catégories qui non seulement délivrerait la partenaire d'un inconfort certain, mais la placerait dans une position avantageuse où elle bénéficierait des avantages cumulés des deux sexes en fonction des besoins, en tout opportunisme, alors que lui, qui refuse d'être un héros, serait plongé dans les affres d'une réduction identitaire.

La symbolique de cette abdication se mesure d'ailleurs à l'aune du contraste avec la quête suivante qui est justement réussie, mais où le narrateur tellement affaibli par un mystérieux malaise et devenu incapable de héler tout taxi, est supplanté par une jeune femme qui en hèle un pour lui, de façon si «volontaire » qu'un véhicule «s'immobilis[e]» aussitôt (105). Par cette passation humoristique des pouvoirs, l'auteur ne cesse d'illustrer à sa façon, à travers la réversibilité des normes de genre, la porosité fondamentale des identités sexuées, passage obligé d'où émerge, dans la douleur, une humanité nouvelle qui doit s'inventer à chaque instant.

L'autre prérogative, sexuelle, est également retirée au narrateur. Déjà dubitatif sur son rôle assigné de dispensateur de plaisir, il est paradoxalement contré dans ses moindres tentatives: chaque fois qu'il pense justement céder à la requête du genre et embrasser Marie, il est débordé sur son propre terrain, renvoyé à son impuissance en se heurtant à sa «bouche fermée, close et butée », ses lèvres «crispées dans la recherche d'un plaisir exclusivement sexuel » $(26,74)$. Quand il cède finalement au deuxième volet de la requête féminine, il est alors victime d'un coïtus interruptus involontaire et loufoque où, coupé dans ses élans par un fax, il se déclare «anéanti par cet incident » (30). Or, plus qu'une panne ponctuelle liée à un simple «incident», il semble que le héros soit placé dès le début de sa relation sous le signe de l'interruption et, déjà, du faux-fuyant : son geste initial de «heurter» son verre à pied contre celui de Marie «dans un simulacre de trinquer sitôt entamé qu'interrompu » était alors perçu par l'intéressée comme un acte de séduction réussi (18). Tout autre est l'interprétation que fait la même destinataire de cette réalité désormais récurrente où l'homme (quoique légitimement empêché) se refuse sexuellement, tout comme il se retient d'embrasser : «elle prit mon interruption pour une agression » (31). Plus qu'une frustration légitime, l'interprétation radicale de Marie se comprend à la lecture d'une inversion des rôles sexués. Et comme en se refusant il fait la femme, elle va tenter de le forcer. 
Poursuivi par cette scène initiale fondatrice et par l'agressivité générée qui le pose doublement en victime, le narrateur se fait voler le plaisir, sans qu'aucune solution véritablement adaptée soit avancée (autre que cette rupture envisagée comme argument compensatoire)... Il se découvre instrument involontaire du plaisir féminin, ce qui suscite son irritation latente dans le roman. «Marie Madeleine Marguerite » se sert de lui de façon répétée comme motif masturbatoire, entretenant les conditions favorables à sa jouissance: au début, par résistance instinctive de l'homme chosifié (elle «se frottait éperdument contre mon pubis à la recherche d'une jouissance que je la sentais prête à conquérir de façon de plus en plus agressive » 29), plus tard parce que le couple est en public et que l'infaisabilité pratique est renforcée par la position debout sur le pont : le narrateur avoue sa «peur » de cette «femme inconnue » «qui se coll[e] » contre lui pour jouir de lui à son insu, et qui est capable de passer outre à la fois aux perturbations cosmiques (« la terre venait de trembler ») et à la pudeur sociale («indifférente aux passants ») (74). Car la secousse sismique a fait renaître brutalement en Marie, - par le besoin d'être rassurée puis, à défaut, celui de se défouler -, un des clichés du genre qui exercent à nouveau sur le narrateur une exigence douloureuse.

D'où la riposte, que l'auteur esquisse à dessein parodique, d'un homme qui ne trouve comme moyen pour se débarrasser d'un double qui exige l'accomplissement de sa mission sociale que de «lui enfon[cer] un doigt» dans un orifice annexe (75): seul exutoire pour sortir d'une confrontation qui engage, par-delà la couple, tous les acteurs sociaux, et renvoie symboliquement le double à sa virilité réelle. Seul hommage apparent et en fait seul défi (à fonction étonnamment ludique, voire ironique) pour se jouer ici de sa partenaire sous couvert de la combler.

Le titre Faire l'amour se pose donc comme violemment antiphrastique puisqu'il n'est question que de ne pas le faire, qu'il y ait chez le narrateur (dans un flou concerté) absence de volonté ou absence de pouvoir. Faire l'amour est le récit d'un homme épuisé, vampirisé. Son imaginaire est en déroute, entre tremblement de terre réel ou fantasmé. Son corps est en souffrance : grippé, il tremble et il «transpir[e] lourdement » (104), «marin[e] dans [s]a sueur » (106), comme si la transpiration ou l'écoulement nasal apportaient une liquidité substitutive.

Nauséeux, il vomit, ou au moins il essaie, car même ce jet hors de lui est difficile : il n'arrive à extirper de lui-même qu'un peu de salive, puis enfin à faire «gicl[er] dans la cuvette » «quelques gouttes» de bile, et encore, en se mettant les doigts dans la gorge, nouvel orifice de substitution (139). Pas de surprise, dans cette oralité déplacée, à ce que le narrateur se refuse au baiser, dans un amour-succion qui lui a déjà soutiré toute son énergie. Il semble à ce point que ce liquide «très aigre » (139) extrait dans la douleur annonce les vertus agressives de l'acide. 
Mais le jet apparaît ici à la fois comme un exutoire à l'impuissance, et un motif actif d'écriture. Car l'étonnant, en l'occurrence, est l'impudeur redondante de l'évocation, pourtant peu flatteuse : le narrateur donne les moindres détails de ce qui pourrait sembler une déchéance, comme si une jouissance nouvelle venait, au niveau de l'écriture, le sortir du cadre traditionnel de la virilité.

Dans ce contexte l'environnement japonais offre un repère à la conscience en déroute, par différents motifs en abyme, matériels ou humains : l'enjeu interculturel extrêmise l'enjeu intersexué auquel il se superpose. Les «haltères » dans la salle de gymnastique de l'hôtel s'offrent ainsi à stimuler les muscles virils et de nuit, tous les accessoires sportifs non utilisés semblent «brisés ou amputés » (38). De même dans le journal c'est avec dérision que le narrateur contemple un modèle du genre ( « une photo du sumotori Musashimaru en super mauvaise posture, putain »120), - alors que lui-même est sur un tatami où il se repose au lieu de s'entraîner. L'environnement ne cesse de lui renvoyer des images de doubles masculins discrédités, que non sans humour il récuse. Parallèlement les accessoires vestimentaires arborés par les collaborateurs japonais de Marie, « engoncés » dans des costumes trois pièces «trop vieux pour eux » (90), sont supposés leur conférer une autorité niée par «l'incurie » de ce «triumvirat» (58). La rue renvoie l'image d'un autre groupe sexué qui est décalqué sur le premier avec, au seuil d'un bar, une femme en minijupe et ciré rose, cliché du genre, et «deux hommes émaciés en costumes trois-pièces » (52); or justement l'attention du narrateur se focalise sur un motif annexe «près des poubelles », qu'il élit en miroir : «la maigre silhouette d'un vieil homme-sandwich dégarni et pensif » (52). Il semble rester rêveur sur cette dualité d'homme pris en «sandwich » qui est ici arborée publiquement, de façon professionnelle, comme seul recours au statut social déclassé.

Les chaussures sont un autre indicateur de controverse sexuée liée à la culture : si le directeur du musée porte des «Puma blanches flamboyantes, avec un fauve stylisé sur chaque pied prêt à bondir » (99), d'une marque occidentale, le narrateur devra suivre la coutume japonaise et quitter les siennes, apprendre à ses dépens la «pénible volte-face » pour se relever après les avoir délacées, et suivre Bernard «en chaussettes sur les marches étroites et glissantes des escaliers » (119). Dans ce Japon voué à l'alternative inconfortable - soit par le fantasme d'agression féline, soit par le risque de chute pour un Occidental (alors que l'Oriental pratique naturellement cette coutume: un voyageur japonais en cravate est focalisé dans le train lisant le journal «en chaussettes » 110) -, Bernard a beau jeu de mettre en garde le narrateur contre l'inversion des règles occidentales dans le domaine routier : «on roule à gauche au Japon, ajouta-t-il, pince-sansrire » (121), prévenant le narrateur sur la nécessité de l'inversion. 
Il est révélateur que certaines pratiques japonaises ravissent le narrateur lorsqu'elles déstabilisent les habitudes occidentales : l'amant mal vertébré n'a aucune difficulté à avaler à la japonaise sa «soupe aux nouilles », alors que Marie n'y parvient pas. Au lieu de faire « remonter les nouilles par paliers avec les baguettes avant de les engloutir bruyamment dans une aspiration précipitée »selon le modèle japonais, elle va «à la pêche aux udons » et «touill[e] mollement sa soupe une baguette dans chaque main » (55); toute action anodine devient le prétexte d'une confrontation plus souterraine et symbolique : car les udons (comme le narrateur) se refusent à Marie, dans un baiser-succion qui l'eût comblée. Au lieu de se laisser aller à la japonaise, de façon plus goulue mais efficace, elle oppose exceptionnellement une réserve occidentale (et traditionnellement féminine). Il est révélateur que son échec non seulement la laisse sur sa faim, mais soit perçu par le narrateur - dans un réjouissement suggestif : elle «faisait peine à voir (ou plaisir, c'était selon)»- en termes d'œuvre d'art inaboutie puisqu'il la compare dans ses vains efforts à « un chef d'orchestre accablé » (ibid.). Usurpant le résultat du cliché décalé (manger à l'occidentale un plat japonais), le narrateur reprend ici l'avantage grâce à la dénonciation culturelle. Non seulement le passage au masculin du chef démotivé montre une accession à l'art possible uniquement pour la femme à travers un changement de genre (grammatical et sexué), mais il évacue Marie de la sphère des compromis délicats. Le masculin de la profession musicale est rendu inopérant, impuissant à la fois dans son expression artistique et dans sa fonction de diriger, sorte de simulacre négatif d'une interaction délétère entre les sexes et les cultures. Ainsi Marie, engoncée elle-même dans sa quête du genre perdu (comme de l'homme viril embrasseur) est-elle exclue d'une performance esthétique où le narrateur s'empresse de la remplacer, non sans fausse ingénuité, et d'assumer enfin le rôle de l'homme barbare et glouton qui sait, lui, sinon déchiqueter sa proie avec les dents, du moins l'avaler tout rond, sans la mâcher. Face à la performance discréditée de l'homme-orchestre, l'homme-boa ne restaure donc sa supériorité de mâle sauvage qu'à travers son accointance pour un motif dérisoire et désopilant (la nouille).

Nulle surprise à ce que Marie ainsi dépouillée réagisse par un sursaut d'appropriation : elle «fouill[e] dans les poches » de son manteau que le narrateur porte toujours, et lui «entourant le corps de ses bras », en sort une cigarette «fripée chancelante et à moitié brisée, avec son nez de Concorde » (56). A défaut de nouilles japonaises donc, informes et glissantes, elle absorbe un organe occidental mieux connu, mais équivoque ici et ayant perdu sa rectitude. Ainsi le narrateur est-il vidé des profits insoupçonnés de son travestissement, et même si cette nouvelle pêche est dérisoire, l'objet exhumé, par sa forme effondrée, devient le symbole d'une science occidentale en état pitoyable, ou du moins en impuissance instable. La métaphore témoigne d'un déplacement des catégories, qui n'empêche néanmoins pas la consommation et la fonction alimentaire substitutive de s'opérer, en parallèle du réconfort culturel. 
On peut s'interroger sur la finalité de ce roman, miroir du monde contemporain sur bien des points comme cette difficile cohabitation entre les sexes, doublée ici d'une confrontation intéressante entre les cultures. Jean-Philippe Toussaint complexifie ses héros en les chargeant volontairement des attributs inversés du genre. Mais il ne tranche pas sur le bien-fondé de leur être. Si Marie incarne une certaine revanche des femmes dans les deux sphères traditionnellement interdites à l'initiative féminine - le professionnel et le sexuel -, elle s'approprie parfois sans nuance les exigences du masculin. Elle n'a pas pris conscience de cette contradiction profonde qui a germé en elle et qu'elle subit, n'ayant pas fait l'unité entre l'ambition intolérante et la dépendance affective. Mais d'un autre côté le masculin ne connaît aucune promotion, même dans sa version libérée du tabou de la domination. Si dans le meurtre final de la fleur, le narrateur semble reprendre le pouvoir et avoir le dernier mot (146), la valeur caricaturale de cet acte sublimatoire n'en est que plus inconsistante, apparemment ridicule, et ne fait que confirmer l'impuissance réelle d'un masculin acculé à l'exutoire. Entre une féminité qui singe le masculin, et une virilité défaillante traversée de féminin et condamnée à se satisfaire d'expédients dérisoires (pour poétiques qu'ils soient), Toussaint ne cesse de rejouer les modalités d'un mal-être nouveau, dès lors que le pouvoir devient interchangeable et n'est plus l'objet d'une répartition prétendument naturelle entre les sexes.

S'il prend tous les clichés à rebours, l'auteur ne gratifie pour autant aucun parti d'une suprématie idéologique : il laisse ses deux héros comme deux enfants encore immatures préfigurant une humanité nouvelle et encore incertaine. Ce n'est que sur le mode ludique qu'il se sort de cet effondrement délibéré des catégories de genre : s'il se plaît à redistribuer les cartes du jeu social, il laisse intelligemment ses lecteurs sur le fil du rasoir, sur une incertitude savoureuse, un inachèvement nécessaire, qui sont les seules issues de l'œuvre littéraire.

- Toutes les références renvoient à l'édition 2014 de Faire l'amour, Editions de minuit, nº 61. Sur l'œuvre de Toussaint :

- Bouju Emmanuel, «Énergie romanesque et reprise d'autorité (Emmanuel Carrère, Noémi Lefebvre, Jean-Philippe Toussaint) », L'Esprit Créateur, vol 54, $\mathrm{n}^{\circ}$ 3, The Idea of Literature / La pensée littéraire (Oana Panaïté dir.), 2014

- Stroh, Olivier, Jean-Philippe Toussaint, Faire l'amour, ArtsLivres, n4ㅌ, 02.V.12 http://artslivres.com/ShowArticle.php?Id=324

- Textyles $n^{\circ} 38$, Jean-Philippe Toussaint (sous la direction de Laurent Demoulin et Pierre Piret), Le Cri/Textyles, 2011.

\footnotetext{
1 Butler, Judith, Trouble dans le genre, La Découverte, 2005, p. 53 et 260.

2 ibid., p. 131-133
} 